\title{
Statistical Learning Techniques and Their Applications for Condition Assessment of Power Transformer
}

\author{
Hui Ma, Tapan K. Saha and Chandima Ekanayake \\ The University of Queensland \\ Brisbane, Australia
}

\begin{abstract}
The condition of power transformers has a significant impact on the reliable operation of the electric power grid. A number of techniques have been in use for condition assessment of transformers. However, interpreting measurement data obtained from these techniques is still a non-trivial task; correlating measurement data to transformer condition is even more difficult. This paper investigates statistical learning techniques, which is able to learn statistical properties of a system from known samples and to predict the system output for unknown samples. Within the statistical learning framework, this paper develops a support vector machine (SVM) algorithm, which can be utilised for automatically analyzing measurement data and assessing condition of transformers. Case studies are presented to demonstrate the applicability of the developed algorithm for condition assessment of power transformer.
\end{abstract}

Index Terms - Condition monitoring, dissolved gas analysis, polarization/depolarization currents, and power transformer.

\section{INTRODUCTION}

POWER transformer is one of the pivotal assets in a power system. Its health condition has a significant impact on the reliable delivery of electricity. However, with substantial growth in demand, transformers have been more frequently loaded than before. Meanwhile a large number of transformers are reaching their designed life or in certain cases operating beyond their designed life. Due to high cost associated with a transformer, it is not always economical to replace it with a new unit by only considering the age of the transformer. As a result, continuously monitoring and effective condition assessment is essential for safe and reliable operation of transformers. Some key issues and methodologies of transformer condition assessment can be found in $[1,2]$.

Over the past decades a number of electrical and chemical techniques have been developed for condition assessment of transformers. Among them polarization and depolarization current (PDC) measurements and dissolved gas analysis (DGA) have attracted much attention across the research communities and utilities. A detailed review of PDC techniques for transformer condition assessment can be found in one of the authors' previous publication [3]. Several industry standards are available for DGA based transformer fault diagnosis, for example the IEEE standard [4]. However, there are still considerable challenges and issues remaining in accurately interpreting measured data and explicitly making inference of a transformer's condition based on the

Manuscript received on 20 August 2011, in final form 27 October 2011. measurements of above electrical and chemical techniques.

The interpretation schemes for PDC reported in the literature are mostly heuristic based and they make condition assessment mainly based on the measurement data acquired from the transformer, which is under investigation $[5,6]$. However, it would be a significant feature that some historical measurement data collected from other transformers can be utilised as references in the process of condition assessment for the transformer under investigation. Especially, a computer program (algorithm) can be trained to acquire the knowledge of underlying statistical dependency between historical data and the conditions of corresponding transformers; such knowledge is then used to assist in evaluating the condition of a present transformer [7]. Moreover, due to imprecision in measurement systems, influence of environmental interference and the limitation of human knowledge, there would be a certain degree of inaccuracy and uncertainty in data interpretation. Therefore, it would be appropriate to estimate the probability distribution in transformer condition assessment to capture above uncertainties [8].

In DGA interpretation, conventional ratio based schemes relate key gas ratios to several predefined conditions of transformers [4, 9]. In recent years, a number of artificial intelligence (AI) techniques have been applied to interpret DGA data including neural network, expert system, fuzzy logic, and self-organizing map [10-12]. In practice, many factors such as operating condition and utility practice have considerable impacts on the AI based algorithms. Therefore, to apply AI algorithms to transformer condition assessment, extensive verifications are required. 
This paper investigates statistical learning techniques to develop an intelligent interpretation scheme for condition assessment of the power transformer. The statistical learning is a data centric approach, which learns unknown dependency between a system's inputs and outputs from the collected data samples. Such dependency is then utilised to make prediction of future system outputs upon receiving new input data. Assuming for a number of transformers, their electrical or chemical measurement data are available and the corresponding conditions of these transformers are known, the statistical learning approach builds a model to approximate the underlying dependency between the measured data and the transformer condition. The model can later be used to make condition assessment for other transformers. Within the statistical learning framework, a support vector machine (SVM) algorithm has been developed in this paper to provide an intelligent tool for automatically analyzing measurement data and making condition assessment of transformers.

The organization of this paper is as follows. Section 2 briefly reviews PDC and DGA techniques. Section 3 discusses the key ideas of the statistical learning technique, and describes the mathematic formulation and implementation of SVM algorithm. Section 4 presents the case studies in which the developed algorithm is applied to make transformer condition assessment based on DGA and PDC measurements. Section 5 concludes finding from this research work.

\section{REVIEW OF PDC AND DGA TECHNIQUES}

\subsection{PDC TECHNIQUES [5]}

In PDC measurement, a step dc voltage $U_{0}$ is applied across the transformer insulation while recording the corresponding current at the transformer terminals. The resultant current due to conduction and slow polarization processes is

$$
I_{p o l}(t)=\left\{\frac{\sigma}{\varepsilon_{0}}+f(t)\right\} C_{0} U_{0} \quad 0<t<t_{0}
$$

where $C_{0}=\frac{C_{m}}{\varepsilon_{r}}$ is the geometrical capacitance of transformer insulation ( $C_{m}$ is the measured capacitance and $\varepsilon_{r}$ is the relative permittivity of transformer insulation at power frequency), $\sigma$ is the average conductivity of the transformer insulation, $\varepsilon_{0}$ is the permittivity of free space, and $f(t)$ is the dielectric response function which is a monotonically decreasing function in time.

At time $t=t_{0}$ the above dc voltage $U_{0}$ is removed and transformer insulation is externally short circuited through an electrometer which records the current. Due to the reorientation of dipoles there will be a depolarization current flowing through the transformer insulation which opposes the polarization direction:

$$
I_{\text {depol }}(t)=-\left\{f(t)-f\left(t+t_{0}\right)\right\} C_{0} U_{0} \quad 0<t<\infty
$$

If the transformer insulation is charged for a sufficiently long time, the effect of $f\left(t+t_{0}\right)$ on equation 2 can be neglected [5]. As a result equation 2 becomes

$$
I_{\text {depol }}(t) \approx-f(t) \times C_{0} U_{0}
$$

Equation 3 implies that the depolarization current can be approximated by deducting the conductivity current (first part in equation 1) from the polarization current given the charging time is sufficiently long.

Dielectric polarization of transformer insulation is a combination of various polarization mechanisms having different response times for the applied electric field. Therefore the electrical response of transformer insulation system can be represented by an electric circuit consisting of resistors and capacitors as shown in Figure 1. In Figure 1 the response of dipoles are represented by a series combination of resistance $R_{i}$ and capacitance $C_{i}$, which have an associated time constant of $\tau_{\mathrm{i}}=R_{i} \times C_{i} ; 1 / R_{0}$ is the dc conduction and $C_{m}$ is the measured capacitance at power frequency.

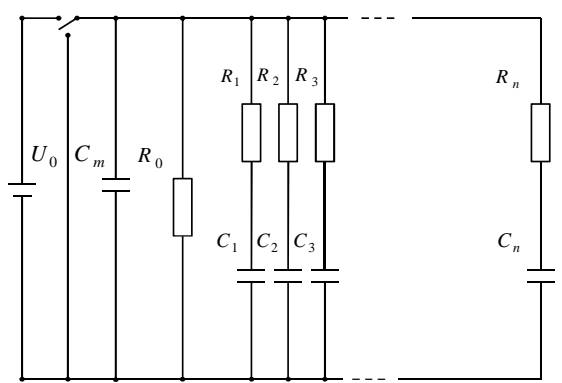

Figure 1. Circuitry representation of transformer insulation system.

An algorithm has been developed by one of the authors of this paper which extracts the above parameters, i.e. the resistances and capacitances from the measured polarization and depolarization currents [13]. In this paper, the extracted parameters will be adopted as discriminative features in statistical learning algorithm for assessing transformer condition based on PDC measurements.

\subsection{DGA TECHNIQUES [4, 9]}

Operating under excessive thermal and electrical stresses, the insulation system of a transformer will form by-product gases such as hydrogen $\left(\mathrm{H}_{2}\right)$, methane $\left(\mathrm{CH}_{4}\right)$, acetylene $\left(\mathrm{C}_{2} \mathrm{H}_{2}\right)$, ethylene $\left(\mathrm{C}_{2} \mathrm{H}_{4}\right)$, ethane $\left(\mathrm{C}_{2} \mathrm{H}_{6}\right)$, carbon monoxide (CO) and carbon dioxide ( $\left.\mathrm{CO}_{2}\right)$. DGA technique makes use of the concentrations or relative proportions of these gases for transformer fault diagnosis. The commonly adopted DGA interpretation schemes include key gas method, ratio based method and artificial intelligence (AI) method.

In the key gas method, several gas contents are adopted to detect four types of faults including oil overheating, paper overheating, partial discharge, and arcing. In the ratio based 
methods such as Dörnenburg Ratios, Rogers Ratios, Duval Triangle and IEC Ratios, the diagnosis is made by computing several key gas ratios and mapping these ratios to the predefined fault patterns.

Although widely adopted in utilities, key gas and ratio based methods still have some limitations. For example, the ratio based schemes may lead to discrepancies in interpretation. Sometimes it may not be able to provide an interpretation for every possible combination of ratio values and give an explicit diagnosis. To address these limitations, artificial intelligence (AI) techniques have been developed to recognize different fault conditions of transformers [10-12]. Different AI techniques have their own merits as well as limitations. There are still avenues for further research, especially the applicability and adaptability of these techniques when they are applied at different utilities.

\section{STATISTICAL LEARNING TECHNIQUE AND ITS ALGORITHM}

\subsection{CONCEPT OF STATISTICAL LEARNING}

Statistical learning algorithm estimates an unknown (input, output) dependency of a system using a set of previously collected and already known samples and upon such estimation it builds up an approximated model which can be subsequently used for predicting future system output upon receiving new input [14-16]. Instead of estimating "true" model (i.e. density estimation), the statistical learning aims to achieve good generalization from the available data. Figure 2 presents the concept and procedure of statistical learning.

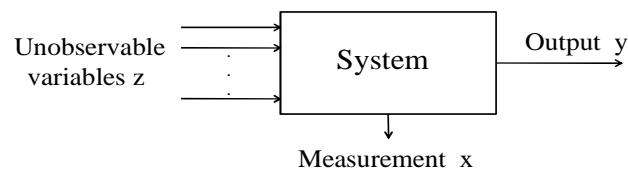

(a)

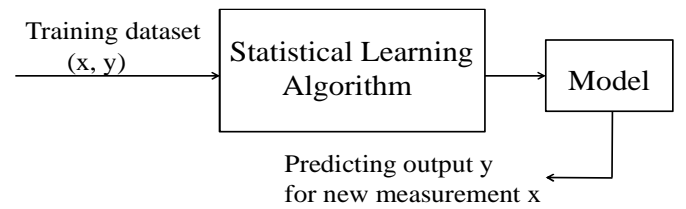

(b)

Figure 2. Illustrative diagrams of statistical learning. (a) Concept of statistical learning; (b) Procedure of statistical learning.

In Figure 2, training data point $(\mathrm{x}, \mathrm{y})$ denotes the already known sample with the measurement (observation) $\mathrm{x}$ and output $\mathrm{y}$. The variables $\mathrm{z}$ denote the factors which have impacts on system outputs but their values are not directly observable. In PDC measurement, the variation of polarization and depolarization currents with time are measurements $\mathrm{x}$ while the condition of the transformer insulation is the system output $\mathrm{y}$. Moreover, the moisture contents and ageing highly influence PDC measurement of transformer insulation; however they are hardly to be measured directly and thus can be regarded as variables $\mathrm{z}$. By using the historical measurement data samples, the statistical learning is tasked to exploit the dependency between the polarization and depolarization currents and the insulation condition of the transformer without the explicit knowledge of moisture contents and ageing extents in the transformer (Figure 2a). Upon such exploitations an approximated model can be built up to associate the measured data with the condition of transformer insulation. Consequently, the model is used to interpret any new PDC data and make assessment of insulation condition for the corresponding transformer (Figure 2b).

The key benefit of statistical learning lies in its capability of utilizing the information contained in historical data to investigate properties about future data. Based on statistical learning methodology, this paper develops a SVM algorithm for transformer condition assessment using DGA and PDC measurements.

\subsection{SUPPORT VECTOR MACHINE ALGORITHM}

Support vector machine (SVM) algorithm involves a tradeoff between the accuracy attained on the training data and the "capacity" of prediction on new data without error [17, 18]. This section briefly describes the formulation and implementation of a SVM algorithm.

Assuming there are $N$ samples $\mathbf{X}=\left[\mathbf{x}_{1}, \ldots, \mathbf{x}_{N}\right]$ and each sample belongs to one of the $T$ independent classes $\left\{y_{1}, \ldots, y_{T}\right\}$, the SVM algorithm is tasked to correctly assign each sample to its corresponding class. SVM algorithm starts with transforming the data samples $\mathbf{X}$ from the original space $\mathfrak{R}^{m}$ to a higher dimensional space $\boldsymbol{\Omega}$ through $\mathbf{h}=\phi(\mathbf{X})$. Then SVM searches for an optimal separation hyperplane in that feature space by solving the following quadratic programming $(\mathrm{QP})$ problem:

$$
\begin{aligned}
\text { Minimize } & \frac{1}{2} \mathbf{w}^{T} \mathbf{w}+C \sum_{k=1}^{N} \xi_{k} \\
\text { Subject to } & y_{k}\left(\mathbf{w} \cdot \mathbf{h}_{k}+b\right) \geq 1-\xi_{k} \quad k=1, \ldots, N \\
\text { and } & \xi_{k} \geq 0 \quad k=1, \ldots, N
\end{aligned}
$$

where $C$ is the regularization parameter which balances the margin maximization and classification violation. By introducing Lagrange multiples $\alpha_{k}$, the above QP problem can be transformed into its dual form [17]:

$$
\begin{aligned}
\text { Maximize } & \sum_{k=1}^{N} \alpha_{k}-\frac{1}{2} \sum_{k=1}^{N} \sum_{j=1}^{N} \alpha_{k} \alpha_{j} y_{k} y_{j} \mathbf{h}_{k} \mathbf{h}_{j} \\
\text { Subject to } & \sum_{k=1}^{N} \alpha_{k} \mathbf{h}_{k}=0 \quad 0 \leq \alpha_{k} \leq C \quad k=1, \ldots, N
\end{aligned}
$$

After obtaining the optimal hyperplane, SVM algorithm can predict the class label for a new sample $\mathbf{X}$ as

$$
y=\operatorname{sgn}\left[\sum_{\mathrm{k}=1}^{N_{\underline{s}}} \alpha_{k} y_{k} \mathbf{h}_{k} \cdot \mathbf{h}+b\right]
$$


where $N_{s}$ is the number of support vectors.

To provide the estimates of uncertainty in transformer condition assessment, the probability that one particular data $\mathbf{x}_{k}$ belongs to one of the $T$ classes is also computed. The detailed implementation of the above SVM algorithm can be found in one of the authors' previous publications [8].

\section{CASE STUDIES}

This section presents case studies to demonstrate the applicability of statistical learning techniques and SVM algorithm for transformer condition assessment based on DGA and PDC measurements. In the algorithmic implementation some software routines from the available toolbox have been adopted with necessary modifications and extensions [19].

\subsection{TRANSFORMER CONDITION ASSESSEMENT BASED ON DGA MEASUREMENT}

The aim of this case study is to recognize normal operating condition and fault conditions occurring in transformers which include discharge fault, lower to medium range thermal fault, high range thermal fault and partial discharge. A database consisting of 390 DGA records provided by a utility company is used for verifying the SVM algorithm developed in this paper. In this database, each DGA record contains key gas concentrations in volume as well as the class label of the corresponding transformer, which is based on the utility's diagnosis results obtained from on-site inspections and utility expert knowledge. The gas concentrations, i.e., $\mathrm{C}_{2} \mathrm{H}_{2}, \mathrm{C}_{2} \mathrm{H}_{4}, \mathrm{C}_{2} \mathrm{H}_{6}, \mathrm{CH}_{4}$, and $\mathrm{H}_{2}$ in volume are used as discriminated features (input) in the algorithm, i.e. the input data is a data array with dimensions of $390 \times 5$. Before running the SVM algorithm, the input data are normalized to $[0,1]$ interval.

The above dataset of 390 DGA records are randomly split into two datasets: a training (validation) dataset consisting of 275 records and a testing dataset consisting of 115 records. The radial-based function (RBF) kernel $K\left(\mathbf{x}_{i}, \mathbf{x}_{j}\right)=e^{-\gamma\left(\left\|\mathbf{x}_{i}-\mathbf{x}_{j}\right\|\right)^{2}}$ is adopted for training the SVM algorithm. A grid search is conducted to decide the optimal values for pairs $(C, \gamma)$ on the training (validation) dataset, where $C$ is the regularization parameter for balancing the margin maximization and classification violation (equations 7 and 8 ) and $\gamma$ is the variance parameter of RBF kernel function. Once the optimal $C$ and $\gamma$ are found, the SVM model is trained using the above training dataset. Tenfold cross validation is also performed on the training dataset. And finally the trained SVM model is applied to assess the condition of transformers in the testing dataset. Each transformer in the testing dataset will be assigned a class label with probability to indicate its condition, i.e. in normal operating condition or in fault conditions.

The above procedure of dataset splitting, training (validation) and testing is repeated ten times. The averaged classification accuracy of tenfold cross validation on training dataset over ten trials is $88.62 \%$ (standard deviation $\pm 1.89 \%$ ). And the averaged classification accuracy on testing dataset averaged over ten trials is $88.61 \%$ (standard deviation $\pm 2.03 \%$ ). Table 1 shows some typical classification results of the SVM algorithm on the testing dataset.

Table 1. SVM Assessment Results on 10 Transformers. [Normal DS MT HT PD] is the probability of each class, where Normal - normal operating condition, DS - discharge fault, MT- lower to medium range thermal fault, HT- high range thermal fault, and PD - partial discharge.

\begin{tabular}{c|c|c|c}
\hline Transformer & $\begin{array}{l}\text { Probability } \\
\text { of Each Class }\end{array}$ & $\begin{array}{c}\text { SVM } \\
\text { Results }\end{array}$ & $\begin{array}{c}\text { On-site } \\
\text { Inspection }\end{array}$ \\
\hline TX1 & {$\left[\begin{array}{lllll}\mathbf{0 . 8 6} & 0.02 & 0.06 & 0.01 & 0.05\end{array}\right]$} & Normal & Normal \\
\hline TX2 & {$\left[\begin{array}{lllll}\mathbf{0 . 8 5} & 0.01 & 0.07 & 0.02 & 0.05\end{array}\right]$} & Normal & Normal \\
\hline TX3 & {$\left[\begin{array}{lllll}0.04 & \mathbf{0 . 6 1} & 0.04 & 0.27 & 0.04\end{array}\right]$} & DS & DS \\
\hline TX4 & {$\left[\begin{array}{lllll}0.25 & 0.02 & \mathbf{0 . 6 7} & 0.02 & 0.04\end{array}\right]$} & MT & MT \\
\hline TX5 & {$\left[\begin{array}{lllll}0 & 0.48 & 0 & \mathbf{0 . 5 2} & 0\end{array}\right]$} & HT & HT \\
\hline TX6 & {$\left[\begin{array}{lllll}0 & 0.04 & 0 & 0.03 & \mathbf{0 . 9 3}\end{array}\right]$} & PD & PD \\
\hline TX7 & {$\left[\begin{array}{lllll}0.07 & 0.04 & 0.04 & 0.01 & \mathbf{0 . 8 4}\end{array}\right]$} & PD & PD \\
\hline TX8 & {$\left[\begin{array}{llllll}\mathbf{0 . 8 0} & 0.01 & 0.15 & 0.01 & 0.03\end{array}\right]$} & Normal & Normal \\
\hline TX9 & {$\left[\begin{array}{lllll}0 & 0.01 & \mathbf{0 . 9 7} & 0.02 & 0.0\end{array}\right]$} & MT & MT \\
\hline TX10 & {$\left[\begin{array}{llllll}0.19 & 0.02 & \mathbf{0 . 7 2} & 0.06 & 0.01\end{array}\right]$} & MT & MT \\
\hline
\end{tabular}

Due to the complexity of the ageing mechanism of insulation material, different fault types might co-exist in one transformer. Thus it would be useful to estimate the probabilities for each type of fault. In Table 1 the SVM algorithm indicates the probability of each fault as a percentage. Such interpretation will be beneficial for understanding of the overall "health status" of a power transformer's insulation system. For example, Transformer TX5 in Table 1 is diagnosed as high temperature thermal fault with $52 \%$ probability; meanwhile the SVM algorithm indicates that this transformer also has a $48 \%$ chance of having a discharge fault.

\subsection{TRANSFORMER CONDITION ASSESSMENT BASED ON PDC MEASUREMENT}

\subsubsection{SCALING SCHEME FOR QUANTIFYING TRANSFORMER INSULATION USING PDC MEASUREMENT}

In PDC measurement, one of the major tasks is to investigate the possible reason for changing the magnitude of polarization and depolarization currents - the likelihood of condition change of paper insulation and/or oil insulation. This would help to understand the correlation between PDC measurement and transformer insulation condition.

To quantify the condition of transformer insulation using PDC measurement it is necessary to model the PDC response of the transformer insulation. As explained in Section 2 the RC circuitry representation (Figure 1) is one of the commonly adopted techniques. In that representation the resistance and capacitance of the two largest RC branches and the conductivity of paper are used to represent the influence of paper insulation and the resistance and capacitance of the two smallest RC branches and the conductivity of oil are used to represent the influence of oil insulation [13]. In order to 
quantify the oil and paper insulation condition of a transformer using the above parameters, a scaling scheme is proposed in this paper, which is based on the assumption that the contribution of different polarization processes toward the magnitude of polarization and depolarization current will be changed with the change of insulation condition. The scaling scheme models different condition of oil and paper insulation as follows:

1. Simulating the condition of oil insulation: As the smallest RC branches represent the oil quality [5], the dielectric response of degraded oil can be represented by introducing a scalar coefficient $\alpha(\alpha=1,2, \ldots$,$) to the resistance and$ capacitance of the two RC branches having lowest time constants as shown in equation 10 . In addition, oil conductivity can also be multiplied by the same coefficient to represent the increase of conductivity. The coefficient adopts the values of $\alpha$ to describe the extent of degradation of oil insulation. The larger the value of $\alpha$, the worse the condition of oil insulation.

$$
\begin{aligned}
R_{-1}^{\prime}=\frac{1}{\alpha} \times R_{-1} & R_{-2}^{\prime}=\frac{1}{\alpha} \times R_{-2} \\
C_{-1}^{\prime}=\alpha \times C_{-1} & C_{-2}^{\prime}=\alpha \times C_{-2} \\
\sigma_{o i l}^{\prime}=\alpha \times \sigma_{o i 1} &
\end{aligned}
$$

2. Simulating the condition of paper insulation: As the largest RC branches represent the paper condition [5], the dielectric response of degraded paper can be represented by introducing a scalar coefficient $\beta(\beta=1,2, \ldots$, etc) to the resistance and capacitance of the two RC branches having highest time constants as shown in equation 11. In addition, paper conductivity can also be multiplied by the same coefficient to represent the increase of conductivity. The coefficient adopts the values of $\beta$ to describe the extent of degradation of paper insulation. The larger the value of $\beta$, the worse the condition of paper insulation.

$$
\begin{aligned}
& R_{1}^{\prime}=\frac{1}{\beta} \times R_{1} \quad R_{2}^{\prime}=\frac{1}{\beta} \times R_{2} \\
& C_{1}^{\prime}=\beta \times C_{1} \quad C_{2}^{\prime}=\beta \times C_{2} \\
& \sigma_{\text {paper }}^{\prime}=\beta \times \sigma_{\text {paper }}
\end{aligned}
$$

In the above equations, subscripts 1 and 2 refer to the two largest RC branches and subscripts -1 and -2 refer to the two smallest RC branches of the equivalent circuit, $\sigma_{p a p e r}$ and $\sigma_{\text {oil }}$ are the conductivities of paper and oil, respectively.

In the PDC case studies throughout this section, a so-called reference transformer will be adopted, on which the above scaling scheme is applied. The process starts with the computation of the representative parameters such as resistances and capacitances of the two largest RC branches, paper conductivity, resistances and capacitances of the two smallest RC branches and oil conductivity of the reference transformer from its PDC measurement. These parameters are then processed according to equations 10 and 11 .

To simulate different insulation conditions for other transformers (named as testing transformer) with reference to that of the reference transformer, in the above process the scaling coefficients $\alpha$ and $\beta$ are assigned different values, which include (1) $\alpha=1,2,3, \ldots$, etc for the cases in which the oil conductivity and the extent of polarization processes in oil insulation is higher than that of the reference transformer; (2) $\beta=1,2,3 \ldots$, etc for the cases in which the paper conductivity and the extent of polarization processes in paper insulation is higher than that of the reference transformer; (3) $\alpha=1, \frac{1}{2}, \frac{1}{3} \ldots$ etc for the cases in which the oil conductivity and the extent of polarization processes in oil insulation is lower than that of the reference transformer; and (4) $\beta=1, \frac{1}{2}, \frac{1}{3} \ldots$ etc for the cases in which the paper conductivity and the extent of the polarization processes in paper insulation is lower than that of the reference transformer. Therefore above four scenarios quantifies the level of testing transformer insulation condition with respect to the reference transformer. Accordingly, a dataset can be generated consisting of a number of data points to describe various insulation conditions. This dataset will be used to train the SVM algorithm. The trained SVM model is then used to assess the oil and paper insulation conditions of testing transformers, which are of interest.

To assess the insulation condition of a testing transformer, the representative parameters of this transformer including RC parameters and oil and paper conductivities are firstly calculated from its PDC measurement. These parameters are then fed into the above trained SVM model, which will be subsequently adopted to assess the insulation condition of this test transformer. By adopting the same reference transformer, the insulation conditions of different testing transformers can be evaluated and compared. This will be exemplified in the following case studies.

\subsubsection{CASE STUDIES ON FIELD TRANSFOEMRS}

Table 2 provides the information of a number of transformers A to E. The PDC measurements were conducted for these transformers in an open substation environment. The tests were carried out at the charging dc voltage of $500 \mathrm{~V}$ or $1000 \mathrm{~V}$ and with $10,000 \mathrm{~s}$ charging and discharging periods. PDC measurements were conducted for transformer B before and after oil reclamation, and the corresponding PDC measurements are denoted as $\mathrm{Bm}_{1}$ and $\mathrm{Bm}_{2}$, respectively. Among the above transformers, transformer A is selected as the reference transformer for assessing the condition of four testing transformers $\mathrm{B}$ to $\mathrm{E}$.

The polarization and depolarization currents of transformers A to $\mathrm{E}$ are plotted in Figures 3 to 5. The measured PDC currents of each transformer were normalized by the corresponding testing voltage and geometric capacitance values. Such normalization provides an equivalent base of geometry and charging voltage for easier comparison between different transformers. From Figures 3 to 5 it can be observed that the magnitudes of polarization and depolarization currents 
of reference transformer $A$ are lower than those of transformers B to E. This reveals that the overall insulation condition of transformer $\mathrm{A}$ is better than the insulation condition of transformers B to E.

Table 2. Transformers Information. $\mathrm{Bm} 1$ and $\mathrm{Bm} 2$ are obtained before and after oil reclamation respectively.

\begin{tabular}{c|c|c|c|c}
\hline \multirow{2}{*}{ Transformer } & $\begin{array}{c}\text { Measurement } \\
\text { ID }\end{array}$ & $\begin{array}{c}\text { Year } \\
\text { of Made }\end{array}$ & $\begin{array}{c}\text { Voltage } \\
(\mathrm{KV})\end{array}$ & $\begin{array}{c}\text { Power } \\
\text { (MVA) }\end{array}$ \\
\hline $\mathrm{A}$ & $\mathrm{A}$ & 1968 & $66 / 11$ & 7 \\
\hline $\mathrm{B}$ & $\mathrm{Bm} 1$ & 1966 & $330 / 132 / 16$ & 100 \\
\cline { 2 - 3 } & $\mathrm{Bm} 2$ & 1966 & $330 / 132 / 16$ & 100 \\
\hline $\mathrm{C}$ & $\mathrm{C}$ & 1966 & $330 / 132 / 16$ & 100 \\
\hline $\mathrm{E}$ & $\mathrm{D}$ & 1936 & $66 / 22$ & 35 \\
\hline
\end{tabular}

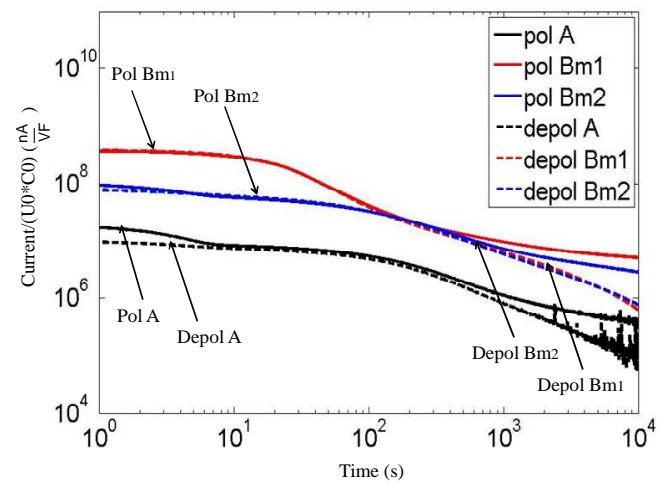

Figure 3. Polarization and depolarization currents obtained from measurements $\mathrm{A}, \mathrm{Bm}_{1}$, and $\mathrm{Bm}_{2}$.

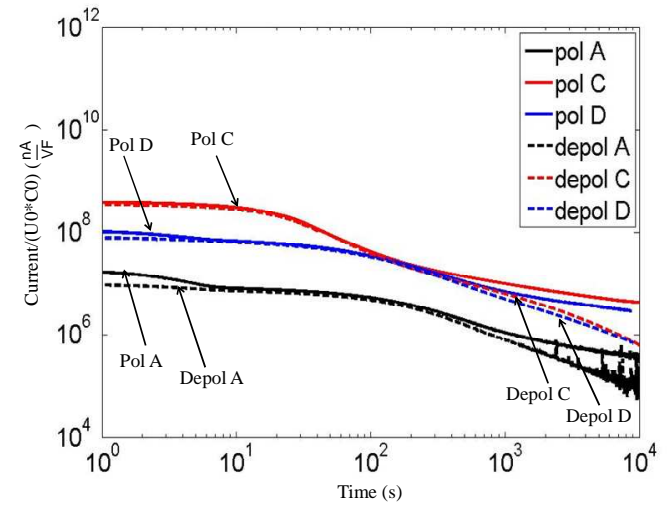

Figure 4. Polarization and depolarization currents obtained from measurements A, C, and D.

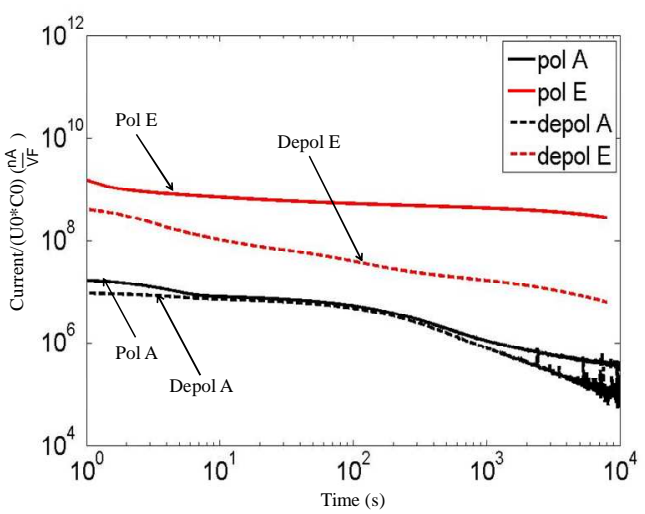

Figure 5. Polarization and depolarization currents obtained from measurements $\mathrm{A}$ and $\mathrm{E}$.
Following the procedure detailed in the previous section, the SVM algorithm is applied to quantify and compare the insulation condition of the above transformers B, C, D, and E with the reference to that of transformer A. The SVM assessment results on insulation conditions of transformers B to $\mathrm{E}$ are summarized in Table 3.

Table 3. SVM Assessment Results for the Insulation Condition of Transformers B to E (With Reference to That of Transformer A).

\begin{tabular}{|c|c|}
\hline Transformer Insulation & $\begin{array}{c}\text { SVM Assessment Results } \\
\text { (with reference to transformer A) }\end{array}$ \\
\hline $\begin{array}{l}\text { Oil of Transformer B } \\
\text { (Before oil reclamation) }\end{array}$ & $\begin{array}{l}\text { About } 90 \% \text { chance of around } 50 \\
\text { times inferior dielectric response }\end{array}$ \\
\hline $\begin{array}{l}\text { Oil of Transformer B } \\
\text { (After oil reclamation) }\end{array}$ & $\begin{array}{l}\text { About } 70 \% \text { chance of around } 5 \\
\text { times inferior dielectric response }\end{array}$ \\
\hline Oil of Transformer C & $\begin{array}{l}\text { About } 90 \% \text { chance of around } 35 \\
\text { times inferior dielectric response }\end{array}$ \\
\hline Oil of Transformer D & $\begin{array}{l}\text { About } 90 \% \text { chance of around } 5 \\
\text { times inferior dielectric response }\end{array}$ \\
\hline Oil of Transformer E & $\begin{array}{l}\text { About } 90 \% \text { chance of around } 50 \\
\text { times inferior dielectric response }\end{array}$ \\
\hline $\begin{array}{l}\text { Paper of transformer B } \\
\text { (Before oil reclamation) }\end{array}$ & $\begin{array}{l}\text { About } 60 \% \text { chance of around } 25 \\
\text { times inferior dielectric response }\end{array}$ \\
\hline $\begin{array}{l}\text { Paper of transformer B } \\
\text { (After oil reclamation) }\end{array}$ & $\begin{array}{l}\text { About } 70 \% \text { chance of around } 10 \\
\text { times inferior dielectric response }\end{array}$ \\
\hline Paper of transformer $\mathrm{C}$ & $\begin{array}{l}\text { About } 70 \% \text { chance of around } 15 \\
\text { times inferior dielectric response }\end{array}$ \\
\hline Paper of transformer D & $\begin{array}{l}\text { About } 70 \% \text { chance of around } 10 \\
\text { times inferior dielectric response }\end{array}$ \\
\hline Paper of transformer E & $\begin{array}{l}\text { About } 90 \% \text { chance of around } 50 \\
\text { times inferior dielectric response }\end{array}$ \\
\hline
\end{tabular}

Amongst the four testing transformers, transformer B had an oil reclamation process carried out on it. It can be seen from Figure 3 that the magnitude of polarization and depolarization current $\mathrm{Bm} 2$, which was measured after the oil reclamation on transformer $\mathrm{B}$ are smaller than that of $\mathrm{Bm} 1$, which was measured before the oil reclamation process. This indicates that the overall insulation of transformer B is improved after oil reclamation. Now the task is to quantify the extent of such insulation condition change. As shown in Table 3, for transformer $\mathrm{B}$, the dielectric response of its oil insulation before the reclamation is around 50 times (with about $90 \%$ probability) inferior to that of the reference transformer A. In contrast, after the oil reclamation, the dielectric response of oil insulation of transformer B is around five times (with about $70 \%$ probability) inferior to that of transformer A. This implies that the oil condition of this transformer is improved about ten times after oil reclamation. As such, the SVM algorithm provides quantitative information regarding oil insulation condition of transformers.

It can also be seen from Table 3 that before oil reclamation, the dielectric response of paper insulation of transformer B is around 25 times (with about $60 \%$ probability) inferior to that of transformer A. However, after oil reclamation, the dielectric response of paper insulation of transformer $\mathrm{B}$ is around 10 times (with about $70 \%$ probability) inferior to that of transformer A. Therefore, it can be concluded that the paper insulation of transformer B is also slightly improved after oil 
reclamation. Condition of paper insulation depends both on ageing process and moisture level. Ageing rate is faster with higher moisture level and vice versa. The oil-reclamation process consisted of filtering and drying the oil through vacuum at high temperature. The dry and filtered oil was pumped back to the tank and the whole process was continued in a closed-cycle. In such a closed-cycle oil reclamation process, it is expected that some moisture and by-products would be driven off the oil. At the same time when the dry oil is circulated through the transformer, some moisture will also migrate out of the paper towards the relatively dry oil. Any measurement carried out after the oil reclamation shows some improvement in paper insulation condition due to low moisture level.

Transformers C and D are both 100 MVA transformers located in the same substation. Transformer $D$ has just undergone oil reclamation before conducting PDC experiments. Table 3 shows that the dielectric response of the oil insulation of $\mathrm{C}$ is around 35 times inferior to that of $\mathrm{A}$; while the dielectric response of the oil insulation of $\mathrm{D}$ is around five times inferior to that that of A. This indicates that the oil condition of $\mathrm{D}$ is better than that of $\mathrm{C}$, and also coincides with the fact that $\mathrm{D}$ was measured just after the oil reclamation. Table 3 also indicates that the condition of paper insulation of $\mathrm{D}$ is slightly better than that of $\mathrm{C}$.

From Figure 5 and Table 3, it can be revealed that the overall insulation condition of transformer $\mathrm{E}$ is more deteriorated, the dielectric response of its oil insulation is about 50 times inferior to that of transformer A. This is not unexpected since transformer $\mathrm{E}$ has been in operation since 1936.

The comparison on insulation conditions can also be made between transformer $\mathrm{B}$ and transformers $\mathrm{C}$ and $\mathrm{D}$. It can be seen from Table 3 that the oil condition of $\mathrm{C}$ is slightly better than that of $\mathrm{B}$ (before oil reclamation): the dielectric response of oil insulation of $\mathrm{C}$ is around 35 times inferior to that of transformer A, while the dielectric response of oil insulation of transformer $\mathrm{B}$ before oil reclamation is around 50 times inferior to that of transformer A. For transformers D and B (after oil reclamation), their oil insulation conditions are almost the same.

The above case study demonstrated that the SVM algorithm can provide quantitative information regarding the condition of paper and oil insulation of a transformer. Through modeling various conditions of oil and paper insulation based on the RC features of a reference transformer the proposed scaling scheme facilitated the condition assessment for other transformers, which are of interest. The next section will provide a case study to reveal the correlation between DGA and PDC measurements by using the SVM algorithm.

\subsection{DGA AND PDC CORRELATION IN TRANSFORMER CONDITION ASSESSMENT}

DGA and PDC technique investigates transformer insulation system from different perspective. In this section the SVM algorithm is applied to make condition assessment on a number of transformers based on both DGA and PDC measurements. It aims to reveal any possible correlation between DGA and PDC measurements on these transformers. Table 4 presents the information of these transformers.

Table 4. Transformers Information.

(Both DGA and PDC Measurements Conducted).

\begin{tabular}{c|c|c|c}
\hline Transformer & $\begin{array}{c}\text { Power } \\
\text { (MVA) }\end{array}$ & Voltage (kV) & Manufacture year \\
\hline D1 & 375 & 330 & 2008 \\
\hline D2 & 133 & 330 & 1974 \\
\hline D3 & 530 & 390 & 1981 \\
\hline D4 & 45 & 23 & 1982 \\
\hline D5 & 45 & 23 & 1982 \\
\hline
\end{tabular}

The SVM model trained in section 4.1 is applied to recognize normal operating and faults conditions for the above five transformers D1 to D5 based on their DGA measurements. The assessment results are shown in Table 5.

Table 5. SVM Assessment Results for Transformers D1 to D5 (DGA). [Normal DS MT HT PD] is the probability of each class, where Normal - normal operating condition, DS - discharge fault, MT- lower to medium range thermal fault, HT- high range thermal fault, and PD - partial discharge.

\begin{tabular}{|c|c|c|c|}
\hline Transformer & & $\begin{array}{c}\text { Probability } \\
\text { of Each Class }\end{array}$ & $\begin{array}{c}\text { SVM } \\
\text { Results }\end{array}$ \\
\hline D1 & {$\left[\begin{array}{ll}0.94 & 0\end{array}\right.$} & $\left.\begin{array}{llll}0 & 0.06 & 0 & 0\end{array}\right]$ & Normal \\
\hline D2 & {$\left[\begin{array}{ll}0.93 & 0\end{array}\right.$} & $\begin{array}{llll}0 & 0.07 & 0 & 0\end{array}$ & Normal \\
\hline D3 & {$\left[\begin{array}{ll}0.88 & 0\end{array}\right.$} & $\left.\begin{array}{llll}0 & 0.12 & 0 & 0\end{array}\right]$ & Normal \\
\hline D4 & {$\left[\begin{array}{ll}0.93 & 0\end{array}\right.$} & $\begin{array}{llll}0 & 0.07 & 0 & 0 \\
\end{array}$ & Normal \\
\hline D5 & {$[0.94 \quad 0$} & $\left.\begin{array}{llll}0 & 0.06 & 0 & 0\end{array}\right]$ & Normal \\
\hline
\end{tabular}

As shown in Table 5, all five transformers D1 to D5 are classified as in normal operation condition. However, compared to other four transformers, D3 has about $12 \%$ chance of having lower to medium range thermal fault. This implies that D3 might be slightly deteriorating toward thermal fault from its normal operating condition. The PDC interpretation results will reveal that the oil insulation of D3 is more degraded than that of other four transformers.

Figure 6 plots the measured polarization currents (normalized by testing voltage and geometric capacitance values) of transformers D1 to D5. The charging dc voltage for these five transformers is $200 \mathrm{~V}$ and the charging period is $10,000 \mathrm{~s}$. The significant current drop of transformer D1 at initial stage might be due to the measurement error.

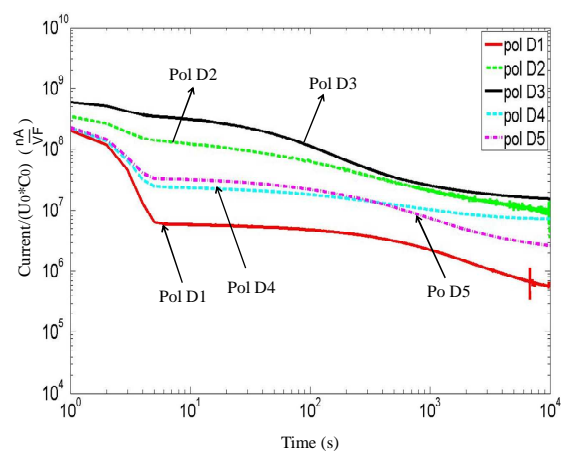

Figure 6. Polarization currents of transformers D1 to D5. 
It can be observed from Figure 6 that the overall polarization current of D1 is lower than that of D2 to D5. This implies that the overall insulation condition of $\mathrm{D} 1$ is better than that of D2, D3, D4 and D5. Therefore, D1 is selected as reference transformer in this case study for assessing the insulation condition of testing transformers D2 to D5.

As discussed in Section 2, the depolarization current can be approximated by deducting the conductivity current from the polarization current. In this case study the approximated depolarization current is used to compute RC parameters for transformers D1 to D5. It is now established that in PDC measurements the initial part of measured current is significantly influenced by the oil insulation while the final part of measured current is influenced by the paper insulation [5]. The SVM algorithm used the resistances and capacitances of the two RC branches with the smallest time constants as the discriminative features for oil insulation, and it used the resistances and capacitances of the two RC branches with the largest time constants as the discriminative features for paper insulation. These parameters were computed from the measured PDC currents. In the computation, the data located around the points with measurements error (Figure 6, the measured currents of Transformer D1) were not necessary. Therefore, the measurement error would not affect the analysis of oil and paper insulation using SVM algorithms.

Adopting the same procedure as detailed in Section 4.2, the scaling scheme is applied to the reference transformer D1 to simulate various oil and paper insulation conditions and generate a dataset to train the SVM algorithm. The trained SVM model is then utilized to make insulation condition assessment on transformers D2 to D5. Table 6 summarized the SVM assessment results for the insulation condition of D2 to D5.

Table 6. SVM Assessment Results for Transformers D2 to D5 (With Reference to Transformer D1).

\begin{tabular}{|c|c|}
\hline Insulation & $\begin{array}{c}\text { Comments } \\
\text { (with reference to transformer D1) }\end{array}$ \\
\hline Oil of Transformer D2 & $\begin{array}{l}\text { About } 60 \% \text { chance of around } 5 \text { times } \\
\text { inferior dielectric response }\end{array}$ \\
\hline Oil of Transformer D3 & $\begin{array}{l}\text { About } 90 \% \text { chance of around } \mathbf{1 5} \text { times } \\
\text { degraded condition }\end{array}$ \\
\hline Oil of Transformer D4 & $\begin{array}{l}\text { About } 70 \% \text { chance of around } 5 \text { times } \\
\text { inferior dielectric response }\end{array}$ \\
\hline Oil of Transformer D5 & $\begin{array}{l}\text { About } 70 \% \text { chance of around } 5 \text { times } \\
\text { inferior dielectric response }\end{array}$ \\
\hline Paper of Transformer D2 & $\begin{array}{l}\text { About } 65 \% \text { chance of around } 5 \text { times } \\
\text { inferior dielectric response }\end{array}$ \\
\hline Paper of Transformer D3 & $\begin{array}{l}\text { About } 70 \% \text { chance of around } \mathbf{1 5} \text { times } \\
\text { inferior dielectric response }\end{array}$ \\
\hline Paper of Transformer D4 & $\begin{array}{l}\text { About } 70 \% \text { chance of around } 5 \text { times } \\
\text { inferior dielectric response }\end{array}$ \\
\hline Paper of Transformer D5 & $\begin{array}{l}\text { About } 65 \% \text { chance of around } 5 \text { times } \\
\text { inferior dielectric response }\end{array}$ \\
\hline
\end{tabular}

As shown in Table 6, the oil condition of D3 is more degraded than that of other three transformers D2, D4, and D5: the dielectric response of oil insulation of transformer D3 is around 15 times inferior to that of $\mathrm{D} 1$; the dielectric response of oil insulation of transformers D2, D4 and D5 are around five times inferior to that of D1. This result coincides with the DGA result as shown in Table 5, which indicates D3 might be slightly deteriorating toward thermal fault while other three transformers are in normal operation condition. Moreover, as shown in Table 6 the SVM classification result also indicates the paper insulation condition of D2, D4, and D5 are better than that of D3.

This case study demonstrated that the SVM algorithm can be successfully used to interpret both DGA and PDC results and can possibly reveal some correlation between these two techniques or can provide complementary information.

\section{CONCULSIONS}

This paper investigated the statistical learning techniques for condition assessment of transformers. Within the statistical learning framework this paper developed an SVM algorithm. The case studies demonstrated the applicability of this algorithm in condition assessment for field transformers. Compared to conventional ratio based DGA interpretation scheme, the algorithm developed in this paper is capable of indicating that several fault conditions may occur simultaneously in a transformer or a transformer might be deteriorating from its normal operating condition by providing probability of each fault condition. The statistical learning technique proposed in this paper provides an effective tool for quantifying the insulation conditions of transformers based on PDC measurement. It makes use of historical PDC data collected from other transformers as reference to train the algorithm for acquiring the knowledge of underlying statistical dependency between historical data and the conditions of corresponding transformer. Such knowledge is then used to assist in evaluating the condition of a transformer of interest.

\section{REFERENCES}

1. M. Wang, A.J. Vandermaar, and K.D. Srivastava, "Review of condition assessment of power transformer in service", IEEE Electr. Insul. Mag., Vol.18, No. 6, pp.12-25, 2002.

2. A.Jahromi, R.Piercy, S.Cress, J.Service, and W.Fan, "An approach to power transformer asset management using health index", IEEE Electr. Insul. Mag., Vol. 25, No. 2, pp. 20-34, 2009

3. T.K. Saha, "Review of modern diagnostic techniques for assessing insulation condition in aged transformers", IEEE Trans. Dielectr. Electr. Insul., Vol. 10, pp. 903-917, 2003.

4. IEEE Guide for the Interpretation of Gases Generated in Oil-Immersed Transformers, IEEE Standard C57.104-2008, 2009.

5. T.K. Saha and P. Purkait, "Investigation of polarisation and depolarisation current measurements for the assessment of oil-paper insulation of aged transformers", IEEE Trans. Dielectr. Electr. Insul., Vol. 11, pp. 144-154, 2004.

6. T. Gradnik, M. Babuder, and M. Koncan-Gradnik, "Estimation of water content in power transformers in service by polarization and depolarization current measurements", IEEE Conf. Dielectr. Liquids, Futuroscope-Chasseneuil, pp.1-4, France, 2008.

7. H. Ma, T. K. Saha, A. Thomas and C. Ekanayake, "Intelligent Framework and Techniques for Power Transformer Insulation Diagnosis", IEEE Power and Energy Society, General Meeting, Paper No. PESGM2009-000425, Calgary, Alberta, Canada, 2009.

8. H. Ma, T.K. Saha, and C. Ekanayake, "Power transformer insulation diagnosis under measurement originated uncertainties", IEEE Power 
and Energy Society, General Meeting, Paper No. 2010GM0299, Minneapolis, Minnesota, USA, 2010.

9. M. Duval, "Dissolved gas analysis: it can save your transformer", IEEE Electr. Insul. Mag., Vol. 5, No.4, pp. 22-27,1989.

10. Z.Y. Wang, Y.L. Liu and P.J. Griffin, "A Combined ANN and expert system tool for transformer fault diagnosis", IEEE Trans. Power Delivery, Vol. 13, pp. 1224-1229, 1998.

11. M. Islam, T. Wu, and G. Ledwich, "A novel fuzzy logic approach to transformer fault diagnosis", IEEE Trans. Dielectr. Electr. Insul., Vol. 7, pp. 177-186, 2000.

12. K.F. Thang, R.K. Aggarwal, A.J. McGrail and D.G. Esp, "Analysis of power transformer dissolved gas data using the self-organizing map", IEEE Trans. Power Delivery, Vol. 18, pp. 1241-1248, 2008.

13. T.K. Saha, P. Purkait, and F. Muller, "Deriving an equivalent circuit of transformers insulation for understanding the dielectric response measurements", IEEE Trans. Power Delivery, Vol. 20, pp.149-157, 2005.

14. V. Cherkassky and F. Mulier, Learning from Data: Concepts, Theory, and Methods, John Wiley \& Sons, Inc., Hoboken, New Jersey, 2007.

15. M.I. Schlesinger and V. Hlavac, Ten Lectures on Statistical and Structural Pattern Recognition, Kluwer Academic Publishers, The Netherlands, 2002.

16. V. Vapnik, The Nature of Statistical Learning Theory, Springer, 1995.

17. C.J.C. Burges, "A tutorial on support vector machines for pattern recognition", Data Mining and Knowledge Discovery, Vol. 2, pp. 121$167,1998$.

18. N. Cristianini and J. Shawe-Taylor, An Introduction of Support Vector Machines and Other Kernel-Based Learning Methods, Cambridge University Press, 2001.

19. C.-C. Chang and C.-J. Lin, "LIBSVM: a library for support vector machines," available: http://www.csie.ntu.edu.tw/ cjlin/libsvm.

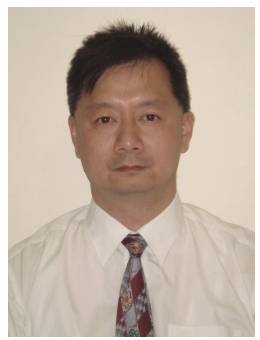

Hui Ma (M'95) received his B.Eng and M.Eng degrees from Xi'an Jiaotong University, China in 1991 and 1994, M.Eng (by research) degree from Nanyang Technological University, Singapore in 1998, and $\mathrm{PhD}$ degree from the University of Adelaide, Adelaide, Australia in 2008. Currently Dr. $\mathrm{Ma}$ is a research fellow in the School of Information Technology and Electrical Engineering, the University of Queensland, Australia. Prior to joining the University of Queensland, Dr. Ma has many years research and development experience. From 1994 to 1995 , he was a researcher in Xi'an Jiaotong University, China. From 1997 to 1999, he worked as a firmware development engineer in CET Technologies Pte. Ltd., Singapore. He was with Singapore Institute of Manufacturing Technology as a research engineer from 1999 to 2003. His research interests include industrial informatics, condition monitoring and diagnosis, power systems, wireless sensor networks, and sensor signal processing.

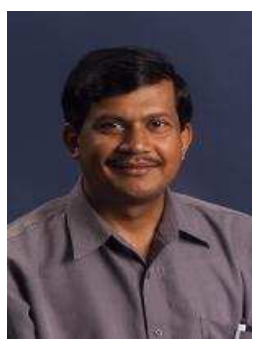

Tapan Kumar Saha (M'93, SM'97) was born in Bangladesh in 1959 and immigrated to Australia in 1989. He received his B. Sc Engineering (electrical and electronic) in 1982 from the Bangladesh University of Engineering \& Technology, Dhaka, Bangladesh, M. Tech (electrical engineering) in1985 from the Indian Institute of Technology, New Delhi, India and PhD in 1994 from the University of Queensland, Brisbane, Australia. Tapan is currently Professor of Electrical Engineering in the School of Information Technology and Electrical Engineering, University of Queensland, Australia. Previously he has had visiting appointments for a semester at both the Royal Institute of Technology (KTH), Stockholm, Sweden and at the University of Newcastle (Australia). He is a Fellow of the Institution of Engineers, Australia. His research interests include condition monitoring of electrical plants, power systems and power quality.

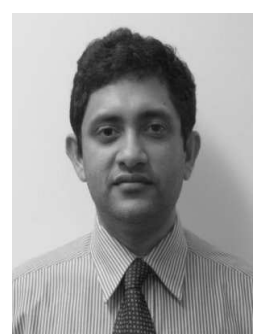

Chandima Ekanayake (M' 00) received his B.Sc.Eng.(Hons) in 1999 from University of Peradeniya Sri Lanka. He obtained his Tech. Lic. and $\mathrm{PhD}$ from Chalmers University of Technology Sweden in 2003 and 2006 respectively. Currently he is a lecturer in the School of Information Technology and Electrical Engineering, the University of Queensland (UQ), Brisbane, Australia. Before joining UQ he was with University of Peradeniya Sri Lanka as a Senior lecturer. During his $\mathrm{PhD}$ studies he was working for a European Union Project called REDIATOOL where he engaged in research related to Diagnostics of Transformer Insulation from dielectric response measurements. From 2001, he has been involving on condition monitoring of transformers installed at Ceylon Electricity Board, Sri Lanka. He was the Chair of IEEE Sri Lanka Section in year 2006 and 2007. His research interests are condition monitoring of power apparatus, alternatives for insulating oil, transient studies on power systems and energy related studies. 